\title{
2 Healthspan and lifespan extension by fecal microbiota transplantation into progeroid mice
}

Clea Bárcena ${ }^{1}$, Rafael Valdés-Mas ${ }^{1}$, Pablo Mayoral ${ }^{1}$, Cecilia Garabaya ${ }^{1}$, Sylvère Durand ${ }^{2,3,4,5}$,

Francisco Rodríguez ${ }^{1}$, María Teresa Fernández-García ${ }^{6}$, Nuria Salazar $^{7,8}$, Alicja M. Nogacka ${ }^{7,8}$, Nuria Garatachea ${ }^{9,10}$, Noélie Bossut ${ }^{2,3,4,5}$, Fanny Aprahamian ${ }^{2,3,4,5}$, Alejandro Lucia ${ }^{11,12}$, Guido

Kroemer $^{2,3,4,5,13,14,15}$, José M. P. Freije ${ }^{1,16}$, Pedro M. Quirós ${ }^{1,16 *}$, and Carlos López-Otín ${ }^{1,16 *}$

${ }^{I}$ Departamento de Bioquímica y Biología Molecular, Facultad de Medicina, Instituto Universitario de Oncología (IUOPA), Universidad de Oviedo, Oviedo, Spain; ${ }^{2}$ Cell Biology and Metabolomics platforms, Gustave Roussy Cancer Campus; Villejuif, France; ${ }^{3}$ Equipe 11 labellisée par la Ligue contre le Cancer, Centre de Recherche des Cordeliers, Paris, France; ${ }^{4}$ INSERM, U1138, Paris, France; ${ }^{5}$ Université Paris Descartes, Sorbonne Paris Cité; Paris, France; ${ }^{6}$ Unidad de histopatología molecular, IUOPA, Universidad de Oviedo, Oviedo, Spain ${ }^{7}$ Department of Microbiology and Biochemistry of Dairy Products, Instituto de Productos Lácteos de Asturias, Consejo Superior de Investigaciones Científicas (IPLA-CSIC), Villaviciosa, Spain; ${ }^{8}$ Diet, Microbiota and Health Group, Instituto de Investigación Sanitaria del Principado de Asturias (ISPA); ${ }^{9}$ Faculty of Health and Sport Sciences, Department of Physiatry and Nursing, University of Zaragoza, Huesca, Spain; ${ }^{10}$ GENUD (Growth, Exercise, NUtrition and Development) Research Group, University of Zaragoza, Zaragoza, Spain; ${ }^{11}$ Faculty of Sport Science, Universidad Europea de Madrid, Madrid, Spain; ${ }^{12}$ Instituto de Investigación Hospital 12 de Octubre $(i+12)$ y Centro de Investigación Biomédica en Red de Fragilidad y Enjevecimiento Saludable (CIBERFES), Spain; ${ }^{13}$ Université Pierre et Marie Curie, Paris, France; ${ }^{14}$ Pôle de Biologie, Hôpital Européen Georges Pompidou, AP-HP; Paris, France; ${ }^{15}$ Karolinska Institute, Department of Women's and Children's Health, Karolinska University Hospital, Stockholm, Sweden; ${ }^{16}$ Centro de Investigación Biomédica en Red de Cáncer (CIBERONC), Spain.

*Send correspondence to:

32 Carlos López-Otín (clo@uniovi.es) or Pedro M. Quirós (pmquiros@gmail.com)

33 Departamento de Bioquímica y Biología Molecular

34 Facultad de Medicina, Universidad de Oviedo

3533006 Oviedo-SPAIN

36 Tel. 34-985-104201; Fax: 34-985-103564 
38 The gut microbiome is emerging as a key regulator of several metabolic, immune and 39 neuroendocrine pathways ${ }^{1,2}$. Gut microbiome deregulation has been implicated in major 40 conditions such as obesity, type-2 diabetes, cardiovascular disease, non-alcoholic fatty

41 acid liver disease and cancer $^{3-6}$, but its precise role in aging remains to be elucidated.

42 Here, we find that two different mouse models of progeria are characterized by intestinal 43 dysbiosis with alterations that include an increase in the abundance of proteobacteria and 44 cyanobacteria, and a decrease in the abundance of verrucomicrobia. Consistent with these 45 findings, we found that human progeria patients also display intestinal dysbiosis and that 46 long-lived humans (i.e., centenarians) exhibit a substantial increase in verrucomicrobia 47 and a reduction in proteobacteria. Fecal microbiota transplantation from wild-type mice 48 enhanced healthspan and lifespan in both progeroid mouse models and transplantation 49 with the verrucomicrobia Akkermansia muciniphila was sufficient to exert beneficial 50 effects. Moreover, metabolomic analysis of ileal content points to the restoration of secondary bile acids as a possible mechanism for the beneficial effects of reestablishing

52 a healthy microbiome. Our results demonstrate that correction of the accelerated aging53 associated intestinal dysbiosis is beneficial, suggesting the existence of a link between 54 aging and the gut microbiota that provides a rationale for microbiome-based interventions against age-related diseases.

58 Keywords: aging, centenarians, fecal transplants, dysbiosis, longevity, metagenomics, 59 microbiome, progeria. 

microbiota have considerably expanded in the last years. It is now known that the microbiota has essential metabolic and immunological functions conserved from worms ${ }^{7}$ to humans ${ }^{1,2}$. In mammals, the gut microbiota is involved in food processing, activation of satiety pathways, protection against pathogens, and production of metabolites including vitamins, short chain fatty acids and secondary bile acids ${ }^{8-10}$. The gut microbiota also signals to distant organs, contributing to the maintenance of host physiology ${ }^{11}$. Intestinal microbiota alterations are associated with major conditions like obesity, type-2 diabetes, cardiovascular disease, non-alcoholic fatty acid liver disease, cancer, and the response to antineoplastic therapy ${ }^{3-6}$.

Although some works have explored the microbiome profile of long-lived humans ${ }^{12,13}$, no alterations have been described in accelerated aging syndromes. In this work, we studied the gut microbiome of two mouse models of Hutchinson-Gilford progeria syndrome (HGPS), patients with HGPS $^{14}$ and Nestor-Guillermo progeria syndrome (NGPS) $)^{15}$, as well as human centenarians and their controls. We found intestinal dysbiosis in both mouse models and progeria patients. In turn, the microbiota of centenarians is characterized by the presence of both pathological- and healthassociated bacterial genera. We show that fecal microbiota transplantation (FMT) from wild-type (WT) donors to progeroid recipients attenuates the accelerated-aging phenotype and increases survival, whereas FMT from progeroid donors to WT recipients

81 induces metabolic alterations. Analysis of centenarians and progeria mouse models point to a beneficial role for the genus Akkermansia, as oral gavage of Akkermansia muciniphila extends the lifespan of progeroid mice.

To explore the relevance of microbiome in progeria, we first studied the gut metagenome profile of the Lmna ${ }^{G 609 G / G 609 G}$ mouse model of $\mathrm{HGPS}^{16}$, by comparing WT 
and $L m n a^{G 609 G / G 609 G}$ mice at three different ages: 1 month (WT 1mo and Lmna ${ }^{G 609 G / G 609 G}$ 1mo), 4 months -when Lmna ${ }^{G 609 G / G 609 G}$ mice exhibit a progeroid phenotype - (WT 4mo and $L m n a^{G 609 G / G 609 G} 4 \mathrm{mo}$ ), and 22 months (for WT mice only; WT 22mo; Extended Data Fig. 1a). To assess how progeria affects the gut microbial community structure, we studied the alpha- and beta-diversity associated with each genotype, and compared the microbial diversity within and between communities. Alpha-diversity was analyzed by calculating the Chao1 (a proxy for community richness) and Shannon's index (a proxy for diversity, taking into account both richness and evenness). We did not observe differences in bacterial diversity or richness within any of the mouse groups (Extended Data Fig. 1b,c and Supplementary Table 1). Next, we evaluated the beta-diversity across mouse groups, identifying a differential clustering of Lmna $6609 G / G 609 G 4$ mo mice in a principal coordinate analysis (PCoA) using the Bray-Curtis dissimilarity (qualitative measure) (Fig 1a) and the Jaccard distances (quantitative measure) (Extended Data Fig. 1d). Similar differences were revealed by hierarchical clustering, where $L m n a^{G 609 G / G 609 G}$ 4mo mice were grouped together and separated from all other groups (Extended Data Fig. 1e,f).

Next, we calculated the percentage of bacterial taxa in each group (Fig. 1b and Extended Data Fig. 2a), applying a linear discriminant analysis (LDA) effect size (LEfSe) method (see Methods; Supplementary Table 2). We noted a similar profile for WT and Lmna ${ }^{G 609 G / G 609 G}$ mice at 1 month of age (Fig. 1 b and Extended Data Fig. 2a), observing solely an increment in the genera Allobaculum from the family Erysipelotrichaceae, Desulfovibrio (class deltaproteobacteria) and Clostridiales of the families Ruminococcaceae and Lachnospiraceae, in Lmna ${ }^{G 609 G / G 609 G} 1$ mo (Extended Data Fig. 2b). As WT mice aged (from 1 to 4 months), we noted an increment in genera such as Allobaculum, Ruminococcus, Coprococcus, Turicibacter or Parabacteroides (Extended 
111 Data Fig. 2c); however, the changes from 1 to 4 months were more profound in progeroid

112 mice, displaying a loss in Akkermansia and Dehalobacterium and an enrichment in

113 Parabacteroides, Prevotella and the Enterobacteriaceae, among other differences

114 (Extended Data Fig. 2d). When comparing WT and progeroid mice at 4 months of age,

115 the pattern was substantially different (Fig. 1b,c and Extended Data Fig. 2a), in agreement

116 with the progression of the aging phenotype. The main differences were a reduction in

117 abundance of Erysipelotrichales (phylum Firmicutes), Burkholderiales (class

118 Betaproteobacteria) and Verrucomicrobiales (phylum Verrucomicrobia) in progeroid

119 mice, together with an increase in Bacteroidales (phylum Bacteroidetes),

120 Deferribacterales (phylum Deferribacteres), YS2 (phylum Cyanobacteria) and the

121 proteobacteria Enterobacteriales and Pseudomonadales (class Gammaproteobacteria)

122 (Fig. 1c and Supplementary Table 2). At a lower taxonomical level, Lmna ${ }^{G 609 G / G 609 G} 4 \mathrm{mo}$

123 mice showed a loss in Clostridiaceae, Allobaculum, Sutterella, Dehalobacterium,

124 Rikenella and Akkermansia (Fig. 1d). Of note, high abundance of Akkermansia has been

125 associated with improved immunomodulation and metabolic homeostasis, reduced

126 inflammation, and protection against atherosclerosis ${ }^{17-19}$. By contrast, $L m n a^{G 609 G / G 609 G}$

$1274 \mathrm{mo}$ exhibited an increment in Mucispirillum, Enterococcus, Acinetobacter,

128 Staphylococcus, Parabacteroides, Bacteroides, Prevotella and Enterobacteriaceae,

129 which contains the genus Escherichia and has been associated with dysbiosis and

130 intestinal inflammation ${ }^{20}$ (Fig. 1d). Changes in Escherichia and Akkermansia were

131 validated afterwards at the species level by qPCR in a second, independent group of

132 Lmna ${ }^{G 609 G / G 609 G}$ mice (Fig. 1e). The gut microbiome of a fraction of WT 22 mo exhibited

133 a tendency towards intestinal dysbiosis (Extended Data Fig. 2a); however, the only

134 significant shift consisted in the loss in Rikenella (Extended Data Fig. 2e), a change also

135 found when comparing Lmna ${ }^{G 609 G / G 609 G} 4$ mo to WT 4 mo mice (Fig. 1d). To explore the 
136 functional implications of the microbiome shift in progeroid mice, we then investigated

137 the metagenome data with PICRUSt and HUMAnN2 (see Methods). LEfSe analysis

138 detected an increase in KEGG pathways related to pathological bacteria such as 'Bacterial

139 invasion of epithelial cells' (ko05100) and 'Flagellar assembly' (ko02040), and a

140 differential enrichment in multiple metabolic pathways (Extended Data Fig. 2f and

141 Supplemental Table S3).

142 To confirm the association of gut dysbiosis with progeria, we analyzed the gut 143 microbiota of a different progeria model, Zmpste24 ${ }^{-/}$mice $^{21}$ (Extended Data Fig. 3a).

144 Although Zmpste24 $4^{-/}$mice showed no differences in bacterial diversity (Extended Data

145 Fig. 3b), they exhibited higher bacterial richness (Extended Data Fig. 3c). Like 146 Lmna ${ }^{G 609 G / G 609 G}$ mice, Zmpste $24^{-/}$animals showed differences in beta diversity at the 147 quantitative (Fig. 1f) and qualitative level (Extended Data Fig. 3d), clustering differently 148 from WT mice, and exhibited signs of dysbiosis (Fig. 1g, Extended Data Fig. 3e) with a 149 high abundance of Proteobacteria (class Alphaproteobacteria) and Cyanobacteria (Fig.

150 1g, Extended Data Fig. 3e and Supplementary Table 2). Zmpste24 ${ }^{-/-}$mice also showed a 151 tendency to a lower amount of Verrucomicrobia (Extended Data Fig. 3e), although this 152 trend was less marked than in $L m n a^{G 609 G / G 609 G}$ animals, perhaps due to the later onset of 153 the progeroid phenotype in Zmpste $24^{-/}$mice. At a lower taxonomic level, we found, 154 among other differences, an enrichment in the order YS2 (phylum Cyanobacteria) and 155 the genera Bacteroides, Parabacteroides and Prevotella (Fig. 1h), similarly to 156 Lmna ${ }^{G 609 G / G 609 G} 4$ mo mice.

157 To investigate the possible existence of gut dysbiosis in human progeria, we 158 obtained fecal samples from four children with HGPS and their healthy siblings, as well 159 as from a patient with NGPS and his healthy sibling and mother (Extended Data Fig. 4a). 160 When comparing progeria patients with their healthy controls, we found no differences 
161 in alpha-diversity (Extended Data Fig. 4b,c) or beta-diversity indices (Fig. 2a and

162 Extended Data Fig. 4d). However, when samples were compared by geographical 163 location (i.e., comparing families), a differential clustering was observed at the 164 quantitative (Fig. 2b) and qualitative levels (Extended Data Fig. 4e). Despite the low 165 number of samples, these results suggest that the environment has more influence on the 166 gut microbiome than the pathological condition, which is in accordance with recent 167 findings ${ }^{22,23}$. Yet, in each of the studied families, the progeria patients had a different 168 profile compared to their healthy siblings. This was particularly evident in families where 169 more than one healthy member was studied (Extended Data Fig. 4f). LEfSe analysis 170 pointed to a loss in Gemmiger -family Ruminococcaceae- and an enrichment in 171 Clostridium from families Erysipelotrichaceae and Lachnospiraceae in progeria patients 172 (Fig. 2c).

173 Considering the gut microbiome alterations observed in mice and progeria 174 patients, we hypothesized that individuals with exceptionally long lifespans (i.e., 175 centenarians) might possess a health-promoting microbiome. Therefore, we performed a 176 metagenomic analysis of a centenarian cohort and ethnically-matched healthy adult 177 controls (Extended Data Fig. 5a). Centenarians showed lower alpha-diversity compared 178 to their controls (Extended Data Fig. 5b), and a considerably lower bacterial richness 179 (Extended Data Fig. 5c). Also, both groups clustered differentially, based on quantitative 180 (Fig. 2d) and qualitative (Extended Data Fig. 5d) beta-diversity indexes, indicating that 181 centenarians had a different microbial community structure than controls. Indeed, we 182 encountered distinct microbial profiles in both groups (Fig. 2e and Extended Data Fig.

183 5e). LEfSe analysis showed that centenarians presented less Betaproteobacteria and more 184 Synergistia and Verrucomicrobiae, among others (Fig. 2e and Supplementary Table 2).

185 At a lower taxonomic level, centenarians were characterized by a lower abundance of 
186 Desulfovibrionaceae, Lachnospiraceae and Erysipelotrichaceae - the last two enriched in 187 progeria patients - and Prevotella, Sutterella, Roseburia, or Butyricicoccus, among other 188 differences (Fig. 2f). Notably, centenarians exhibited a higher abundance of

189 Enterobacteriaceae, Ruminococcaceae and Christensenellaceae and the genera

190 Klebsiella, Lactobacillus, Parabacteroides and Akkermansia (Fig. 2f). These results are

191 in line with previous studies reporting high levels of Akkermansia and

192 Christensenellaceae and low levels of Roseburia in centenarians ${ }^{12,24}$.

193 Based on the aforementioned results, we hypothesized that changes in the gut

194 microbiota might accompany the accelerated aging of HGPS mice. To explore this 195 possibility, we performed FMT in four different mouse groups. First, we used WT control 196 mice as microbiota donors (herein referred to as WTmic, WT microbiota) and 197 Lmna ${ }^{G 609 G / G 609 G}$ mice as recipients. We also transplanted Lmna ${ }^{G 609 G / G 609 G}$ mice with 198 microbiota from older $L m n a^{G 609 G / G 609 G}$ mice, that is, progeroid mice with a more advanced 199 phenotype (oG609Gmic, old Lmna ${ }^{G 609 G / G 609 G}$ microbiota) (Fig. 3a). FMT effectiveness 200 was evaluated by comparing the gut metagenomic profiles of control, transplanted and 201 donor mice, showing that transplanted progeroid mice acquired the donor microbiota 202 (Extended Data Fig. 6a,b). Additionally, we performed microbiome ablation and shamtransplanted Lmna ${ }^{G 609 G / G 609 G}$ mice (herein referred to as EmptyT, empty transplant) (Fig. 3a). Lmna ${ }^{G 609 G / G 609 G}$-WTmic manifested a delayed loss of body weight and temperature (Fig. 3b,c). Phenotype-dependent hypoglycemia was avoided and renal perivascular 206 fibrosis was attenuated in $L m n a^{G 609 G / G 609 G}$-WTmic (Fig. 3d,e). Spleen weight, typically 207 reduced in progeroid mice, was similar in Lmna ${ }^{G 609 G / G 609 G}$-WTmic and Lmna ${ }^{G 609 G / G 609 G}$ 208 controls, but lower in Lmna ${ }^{G 609 G / G 609 G}$-oG609Gmic (Extended Data Fig. 6c). We also 209 noted an increase in intestinal inflammation markers in $L m n a^{G 609 G / G 609 G}$ mice that was 210 recovered in Lmna ${ }^{G 609 G / G 609 G}$-WTmic (Extended Data Fig. 6d). Surprisingly, some 


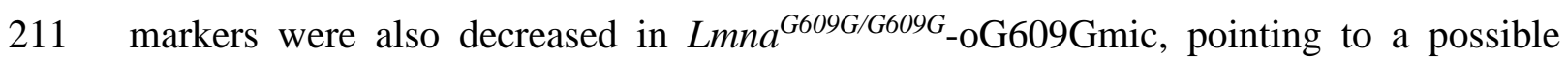

212 beneficial effect of the FMT protocol per se (Extended Data Fig. 6d). Most importantly,

213 Lmna ${ }^{G 609 G / G 609 G}$-WTmic showed improved survival compared to control Lmna G609G/G609G

214 mice $(P=0.0029)$, with a $13.5 \%$ increase in median lifespan (160 vs 141 days,

215 respectively) (Fig. 3f). Lmna ${ }^{G 609 G / G 609 G}$-WTmic also exhibited an extended maximal

216 survival (9\% increment, $P=0.04$ ) (Fig. 3f and Extended Data Fig. 6e). In contrast,

217 Lmna ${ }^{G 609 G / G 609 G}$-oG609Gmic showed reduced survival compared to control

$218 L m n a^{G 609 G / G 609 G}$ mice $(P=0.045)$, with a reduction in median lifespan (129 days) (Fig.

219 3f). Lmna $a^{G 69 G / G 609}$-EmptyT did not show survival differences when compared to control

220 Lmna ${ }^{G 609 G / G 609 G}$ mice (Extended Data Fig. 6f). Lastly, to evaluate the potential

221 pathogenicity of progeroid microbiota, we also performed FMT from old $L m n a^{G 609 G / G 609 G}$

222 donors into WT mice (WT-oG609Gmic). Effective gut colonization was validated by

223 comparing the metagenomic profiles of control, transplanted and donor mice (Extended

224 Data Fig. 6a,b). We did not observe progeria-related features in WT-oG609Gmic mice.

225 However, this maneuver caused metabolic alterations, including a higher body weight,

226 higher glucose levels, lower $\mathrm{O}_{2}$ consumption, lower $\mathrm{CO}_{2}$ production and reduced energy

227 expenditure (Fig. 3g-k and Extended Data Fig. 7a-d).

228 To validate these results, we also performed FMT in Zmpste24 ${ }^{-/}$mice using 229 microbiota from WT mice (Zmpste $24^{-/}$-WTmic). This manipulation caused Zmpste $24^{-/}$

230 mice to manifest a less pronounced cervicothoracic lordokyphosis, larger body size and

231 maintained grooming (Fig. 31). Body weight loss (Fig. 3m and Extended Data Fig. 8a)

232 and hypoglycemia (Fig. 3n) were avoided in Zmpste24 ${ }^{-\%}$-WTmic mice. Like

233 Lmna ${ }^{G 609 G / G 609 G}$ mice transplanted with WT microbiota, Zmpste $24^{-\%}$-WTmic mice also

234 exhibited enhanced survival $(P=0.0092)$, with a median lifespan increase of $13.4 \%(279$ 
vs 246 days) (Fig. 3o). Maximum survival was increased by almost $8 \%(P=0.09)$ (Fig.

23630 and Extended Data Fig. 8b).

The results above in both human and murine gut metagenomic profiles described

238 a lost in A. muciniphila in Lmna ${ }^{G 609 G / G 609 G} 4 \mathrm{mo}$ mice and an increase in centenarians'

239 samples. Since this bacterium exerts beneficial effects in other models ${ }^{17,19,25,26}$, we tested

240 if external supply of A. muciniphila to $L m n a^{G 609 G / G 609 G}$ mice would improve their

241 healthspan or lifespan. By supplementing Lmna $6609 G / G 609 G$ mice with A. muciniphila

242 through oral gavage, we obtained a modest lifespan extension $(P=0.016)$ (Fig. $4 \mathrm{a})$,

243 suggesting a protective role of this microorganism against accelerated aging

244 manifestations. As previously described ${ }^{19}$, A. muciniphila supplementation induced ileal

245 expression of $\operatorname{Reg} 3 g$ (Fig. 4b) and favored the thickening of the intestinal mucosa layer

246 (Fig. 4c). Lmna ${ }^{G 609 G / G 609 G}$ mice receiving A. muciniphila also showed an increment in the

247 intestinal trefoil factor Tff3 (Fig. 4b), which might promote wound healing and repair of

248 the mucosa layer ${ }^{27}$.

249 Finally, to investigate the potential mechanisms accounting for the healthspan and

250 lifespan extension of $L m n a^{G 609 G / G 609 G}$ mice after FMT, we performed metabolome

251 profiling of ileal content from WT, Lmna ${ }^{G 609 G / G 609 G}$ and $L m n a^{G 609 G / G 609 G}$-WTmic mice.

252 Significant changes were analyzed by metabolite set enrichment analysis using KEGG

253 pathways, detecting an enrichment in 'secondary bile acid biosynthesis' (Fig. 4d). As

254 recently described ${ }^{28}$, different bile acids were decreased in $L^{2 m n a^{G 609 G / G 609 G}}$ compared to

255 WT mice, and recovered in $L m n a^{G 609 G / G 609 G}$-WTmic (Fig. 4e). Moreover, Lmna ${ }^{G 609 G / G 609 G}$

256 mice ileal content exhibited a depletion of the monosaccharides arabinose and ribose, the

257 nucleoside inosine, and the ether-phospholipid PCae (18:0) that was reversed upon FMT

258 with WT microbiota (Fig. 4f). 
FMT might modulate obesity and metabolism in humans and mice ${ }^{29,30}$, ameliorate

260 metabolic syndrome in patients ${ }^{31}$, and contribute to the treatment of refractory immune 261 checkpoint inhibitor (ICI)-associated colitis ${ }^{32}$ and recurrent infections by Clostridium difficile ${ }^{33}$. The promising in vivo results obtained in this study suggest that therapeutic interventions on the intestinal microbiome may lead to healthspan end even lifespan

264 improvements. In this regard, we show that A. muciniphila administration lead to a

265 lifespan enhancement in progeria, thus extending previous findings on pro-health activities of Akkermansia spp. in the intestinal tract of mammals ${ }^{17-19}$ and in aged mice ${ }^{34}$.

267 Previous studies have described that successful FMT treatment of recurrent infection by C. difficile relies on restoration of correct bile acid metabolism ${ }^{35,36}$, and that antibioticinduced microbiome depletion reduces the secondary bile acid pool ${ }^{37}$. Of note, secondary bile acids are produced by the gut microbiota ${ }^{38}$ and positively correlate with Akkermansia levels in mice ${ }^{39}$. Considering that bile acids regulate metabolism and anti-inflammatory signals $^{40}$ and that they are depleted in $L m n a^{G 609 G / G 609 G}$ mice $^{28}$, the restoration of secondary bile acids - and other metabolites (arabinose, ribose, inosine) - by FMT might

274 contribute to extend healthspan and lifespan in progeroid mice (Fig. 4g). Future work 275 might identify the functional mechanisms by which some bacterial species and metabolites are responsible for the healthspan and lifespan extension conferred by FMT, and explore the applicability of FMT in normal or accelerated aging. Acknowledgements. We thank M. Stamsnijder from the Progeria Family Circle, and the 280 families that have kindly participated in our study. We also thank G. Velasco, Y. Español, 281 A.R. Folgueras, X.M. Caravia, J.M. Fraile, I. Varela, M. Mittelbrunn and K. Iribarren for 282 helpful comments and advice; R. Feijoo, A. Moyano, D.A. Puente, S.A. Miranda, M.S. 283 Pitiot, V. García de la Fuente and M.C. Muñiz for excellent technical assistance; M. 284 Gueimonde and C.G. de los Reyes-Gavilán (IPLA-CSIC) for providing A. muciniphila; 
and C. Mayolas for help with logistic organization. We also acknowledge the generous support by J.I. Cabrera. A.L. research on aging is funded by Fondo de Investigaciones Sanitarias and Fondos FEDER (PI15/00558). Metabolomics platform is supported by H2020 European Union project OncoBiome. G.K. is supported by the Ligue contre le Cancer (équipe labellisée); Agence National de la Recherche (ANR) - Projets blancs; ANR under the frame of E-Rare-2, the ERA-Net for Research on Rare Diseases; Association pour la recherche sur le cancer (ARC); Cancéropôle Ile-de-France; Chancelerie des universités de Paris (Legs Poix), Fondation pour la Recherche Médicale (FRM); a donation by Elior; European Research Area Network on Cardiovascular Diseases (ERA-CVD, MINOTAUR); Gustave Roussy Odyssea; the European Union Horizon 2020 Project Oncobiome; Fondation Carrefour; Institut National du Cancer (INCa); Inserm (HTE); Institut Universitaire de France; LeDucq Foundation; the LabEx Immuno-Oncology; the RHU Torino Lumière; the Seerave Foundation; the SIRIC Stratified Oncology Cell DNA Repair and Tumor Immune Elimination (SOCRATE); and the SIRIC Cancer Research and Personalized Medicine (CARPEM). The Instituto Universitario de Oncología is supported by Fundación Bancaria Caja de Ahorros de Asturias. J.M.P.F. is supported by Ministerio de Economía y Competitividad-FEDER and Gobierno del Principado de Asturias. C.L-O. is supported by grants from European Research Council (DeAge, ERC Advanced Grant), Ministerio de Economía y Competitividad-FEDER, Instituto de Salud Carlos III (RTICC) and Progeria Research Foundation.

Author contributions. C.B., J.M.P.F., P.M.Q. and C.L.-O. conceived and designed experiments. R.V.-M., P.M.Q. and C.B. performed bioinformatics analysis and results interpretation. C.B., P.M., C.G., F.R. and P.M.Q. performed experiments and analyzed data. M.T.F.-G. performed histopathological analysis. N.S. and A.M.N. performed the culturing and bacterial solutions of A. muciniphila. N.G. and A.L. organized and carried out pickup of human centenarian samples. S.D., N.B., F.A. and G.K. performed LC-MS-

313 based metabolomics. C.B., J.M.P.F., P.M.Q. and C.L.-O. wrote the manuscript. A.L. and

314 G.K. assisted with manuscript edition and all authors revised the manuscript and provided 315 input. 
318 Competing interests statement. GK is one of the scientific co-founders of everImmune.

319

320

321

322

323

324

325

326

327

328

329

330

331

332

333

334

335

336

337

338

339

340

341

342

343

344

345

346

347

348

349

350

351

\section{References}

1. Thaiss, C.A., Zmora, N., Levy, M. \& Elinav, E. The microbiome and innate immunity. Nature 535, 65-74 (2016).

2. Leulier, F., et al. Integrative physiology: at the crossroads of nutrition, microbiota, animal physiology, and human health. Cell Metab 25, 522-534 (2017).

3. Koeth, R.A., et al. Intestinal microbiota metabolism of L-carnitine, a nutrient in red meat, promotes atherosclerosis. Nat Med 19, 576-585 (2013).

4. Loomba, R., et al. Gut microbiome-based metagenomic signature for noninvasive detection of advanced fibrosis in human nonalcoholic fatty liver disease. Cell Metab 25, 1054-1062 e1055 (2017).

5. Qin, J., et al. A metagenome-wide association study of gut microbiota in type 2 diabetes. Nature 490, 55-60 (2012).

6. Zitvogel, L., Daillere, R., Roberti, M.P., Routy, B. \& Kroemer, G. Anticancer effects of the microbiome and its products. Nat Rev Microbiol 15, 465-478 (2017).

7. Cabreiro, F. \& Gems, D. Worms need microbes too: microbiota, health and aging in Caenorhabditis elegans. EMBO Mol Med 5, 1300-1310 (2013).

8. Rios-Covian, D., et al. Intestinal short chain fatty acids and their link with diet and human health. Front Microbiol 7, 185 (2016).

9. Breton, J., et al. Gut commensal E. coli proteins activate host satiety pathways following nutrient-induced bacterial growth. Cell Metab 23, 324-334 (2016).

10. Wahlstrom, A., Sayin, S.I., Marschall, H.U. \& Backhed, F. Intestinal crosstalk between bile acids and microbiota and its impact on host metabolism. Cell Metab 24, 41-50 (2016).

11. Schroeder, B.O. \& Backhed, F. Signals from the gut microbiota to distant organs in physiology and disease. Nat Med 22, 1079-1089 (2016).

12. Biagi, E., et al. Gut microbiota and extreme longevity. Curr Biol 26, 1480-1485 (2016).

13. O'Toole, P.W. \& Jeffery, I.B. Gut microbiota and aging. Science 350, 1214-1215 (2015).

14. Gordon, L.B., Rothman, F.G., Lopez-Otin, C. \& Misteli, T. Progeria: a paradigm for translational medicine. Cell 156, 400-407 (2014). 
15. Puente, X.S., et al. Exome sequencing and functional analysis identifies BANF1 mutation as the cause of a hereditary progeroid syndrome. Am J Hum Genet $\mathbf{8 8}$, 650656 (2011).

16. Osorio, F.G., et al. Splicing-directed therapy in a new mouse model of human accelerated aging. Science translational medicine 3, $106 \mathrm{ra107}$ (2011).

17. Plovier, H., et al. A purified membrane protein from Akkermansia muciniphila or the pasteurized bacterium improves metabolism in obese and diabetic mice. Nat Med 23, 107-113 (2017).

18. Schneeberger, M., et al. Akkermansia muciniphila inversely correlates with the onset of inflammation, altered adipose tissue metabolism and metabolic disorders during obesity in mice. Sci Rep 5, 16643 (2015).

19. Everard, A., et al. Cross-talk between Akkermansia muciniphila and intestinal epithelium controls diet-induced obesity. Proc Natl Acad Sci U S A 110, 9066-9071 (2013).

20. Maharshak, N., et al. Altered enteric microbiota ecology in interleukin 10deficient mice during development and progression of intestinal inflammation. Gut Microbes 4, 316-324 (2013).

21. Varela, I., et al. Accelerated ageing in mice deficient in Zmpste24 protease is linked to p53 signalling activation. Nature 437, 564-568 (2005).

22. Rothschild, D., et al. Environment dominates over host genetics in shaping human gut microbiota. Nature 555, 210-215 (2018).

23. Lloyd-Price, J., et al. Strains, functions and dynamics in the expanded Human Microbiome Project. Nature 550, 61-66 (2017).

24. Goodrich, J.K., et al. Human genetics shape the gut microbiome. Cell 159, 789799 (2014).

25. Gopalakrishnan, V., et al. Gut microbiome modulates response to anti-PD-1 immunotherapy in melanoma patients. Science 359, 97-103 (2018).

26. Routy, B., et al. Gut microbiome influences efficacy of PD-1-based immunotherapy against epithelial tumors. Science 359, $91-97$ (2018).

27. Kalabis, J., Rosenberg, I. \& Podolsky, D.K. Vangl1 protein acts as a downstream effector of intestinal trefoil factor (ITF)/TFF3 signaling and regulates wound healing of intestinal epithelium. J Biol Chem 281, 6434-6441 (2006).

28. Barcena, C., et al. Methionine restriction extends lifespan in progeroid mice and alters lipid and bile acid metabolism. Cell Rep 24, 2392-2403 (2018).

29. Ridaura, V.K., et al. Gut microbiota from twins discordant for obesity modulate metabolism in mice. Science 341, 1241214 (2013). 
30. Cox, L.M., et al. Altering the intestinal microbiota during a critical developmental window has lasting metabolic consequences. Cell 158, 705-721 (2014).

390 31. Vrieze, A., et al. Transfer of intestinal microbiota from lean donors increases 391 insulin sensitivity in individuals with metabolic syndrome. Gastroenterology 143, 913392916 e917 (2012).

393 32. Wang, Y., et al. Fecal microbiota transplantation for refractory immune 394 checkpoint inhibitor-associated colitis. Nat Med 24, 1804-1808 (2018).

395 33. van Nood, E., et al. Duodenal infusion of donor feces for recurrent Clostridium 396 difficile. $N$ Engl J Med 368, 407-415 (2013).

397 34. Bodogai, M., et al. Commensal bacteria contribute to insulin resistance in aging 398 by activating innate B1a cells. Science translational medicine 10(2018).

399 35. Weingarden, A.R., et al. Microbiota transplantation restores normal fecal bile 400 acid composition in recurrent Clostridium difficile infection. Am J Physiol Gastrointest 401 Liver Physiol 306, G310-319 (2014).

402 36. Buffie, C.G., et al. Precision microbiome reconstitution restores bile acid 403 mediated resistance to Clostridium difficile. Nature 517, 205-208 (2015).

404 37. Zarrinpar, A., et al. Antibiotic-induced microbiome depletion alters metabolic 405 homeostasis by affecting gut signaling and colonic metabolism. Nat Commun 9, 2872 406 (2018).

407 38. de Aguiar Vallim, T.Q., Tarling, E.J. \& Edwards, P.A. Pleiotropic roles of bile 408 acids in metabolism. Cell Metab 17, 657-669 (2013).

409 39. Pierre, J.F., et al. Activation of bile acid signaling improves metabolic 410 phenotypes in high-fat diet-induced obese mice. Am J Physiol Gastrointest Liver 411 Physiol 311, G286-304 (2016).

412 40. Postler, T.S. \& Ghosh, S. Understanding the holobiont: how microbial 413 metabolites affect human health and shape the immune system. Cell Metab 26, 110-130 414 (2017). 
423 Figure 1. Gut dysbiosis in $\mathrm{Lmna}^{\mathrm{G} 609 \mathrm{G} / \mathrm{G} 609 \mathrm{G}}$ and $\mathrm{Zmpste24}^{-/-}$progeroid mice. (a) 424 Principal coordinates analysis (PCoA) of beta-diversity using the Bray-Curtis 425 dissimilarity metric among samples of the five groups of mice analyzed $(P=0.001$; 426 PERMANOVA). G609G 4mo mice show statistical differences with each of the other 427 groups (see Supplementary Table 1). Each dot represents an individual mouse. PCo1 428 and PCo2 represent the percentage of variance explained by each coordinate (WT 1mo, $\mathrm{n}=6$; G609G 1mo, $\mathrm{n}=5$; WT 4mo, $\mathrm{n}=8$; G609G 4mo, $\mathrm{n}=9$; WT 22mo, $\mathrm{n}=8$ ). (b)

430 Average relative abundance of prevalent microbiota at the class level in the 5 groups studied: 1-month-old wild-type (WT 1mo, n = 6), 1-month-old Lmna G609G/G609G (G609G 1mo, $\mathrm{n}=5$ ), adult 4-month-old wild-type (WT 4mo, n = 8), 4-month-old Lmna ${ }^{G 609 G / G 609 G}$ (G609G 4mo, $\mathrm{n}=9$ ) and 22-month-old wild-type (WT 22mo, $\mathrm{n}=8$ ). The low abundance bacteria group includes all bacterial classes with less than $0.5 \%$ of total abundance. (c) Taxonomic cladogram obtained from LEfSe analysis showing bacterial taxa (phylum, class and order) that were differentially abundant in progeroid and WT mice at 4 months of age. Red indicates increased abundance in progeroid mice, grey indicates increased abundance in WT mice. Def: Deferribacteres (phylum), Deferribacteres (order), Deferribacterales (class); Cya: Cyanobacteria (phylum); Pseudo: Pseudomonadales; Entero: Enterobacteriales; Burkho: Burkholderiales. (d) Results of LEfSe analysis showing bacterial taxa that were significantly different in abundance between G609G and WT mice at 4 months of age. For c and d: WT 4mo, n = 8; G609G 4mo, n = 9. (e)

443 Validation using qPCR of the differences in abundance of E. coli $(\mathrm{W}=6, P=0.0046)$ and A. muciniphila $(\mathrm{W}=52, P=0.03)$ between WT $(\mathrm{n}=8)$ and G609G mice $(\mathrm{n}=8)$ at 10 weeks of age. Two-tailed unpaired Wilcoxon rank-sum test. Each dot represents an individual mouse. In the box plots, upper and lower hinges correspond to the first and third quartiles, center line represents the median, whiskers indicate the highest and lowest values that are within $1.5 * \mathrm{IQR}$, and data beyond the end of the whiskers are outliers and plotted as points. (f) PCoA of beta-diversity using the Bray-Curtis dissimilarity metric between Zmpste $24^{-/}$and WT mice ( $P=0.003$, PERMANOVA). Each dot represents an individual mouse. PCo1 and PCo2 represent the percentage of variance explained by each coordinate. (g) Average relative abundance of prevalent microbiota at the class level in WT and Zmpste $24^{-/}$mice. (h). LEfSe analysis showing bacterial classes that were significantly different in abundance between Zmpste $24^{-/-}$and WT mice. For f-h: 4-month- 
old WT mice, $\mathrm{n}=8$ (same as panel a-d); 4-month-old Zmpste24 $4^{-/}$mice, $\mathrm{n}=4$. p: phylum; c: class; o: order; f: family; g: genus.

Figure 2. Alterations in the gut microbiome in progeria patients and long-lived

humans. (a,b) PCoA of beta-diversity using the Bray-Curtis dissimilarity metric among fecal samples analyzed by $(\mathbf{a})$ health status $(P=0.5$, PERMANOVA) (Control, $\mathrm{n}=9$ individuals; Progeria, $\mathrm{n}=5$ individuals) and (b) family $(P=0.001$, PERMANOVA) (Family A, n = 3; Family B, $n=3$; Family C, $n=3$; Family D, $n=2$; Family E, $n=3$ ). Each dot represents one person. PCo1 and PCo2 represent the percentage of variance explained by each coordinate. (c). Results of LEfSe analysis showing bacterial genera whose abundance significantly differed between progeria patients and their healthy siblings (Control=9 individuals; Progeria $=5$ individuals). (d) PCoA of beta-diversity using the Bray-Curtis dissimilarity metric of healthy controls ( $\mathrm{HC} ; \mathrm{n}=14$ individuals) and centenarians (Ce; $\mathrm{n}=17$ individuals) $(P=0.001$, PERMANOVA). Each dot represents one person. (e) Average relative abundance of prevalent microbiota at the class level in centenarians (Ce; $\mathrm{n}=17$ individuals) and healthy controls ( $\mathrm{HC} ; \mathrm{n}=14$ individuals). The low abundance bacteria group includes all bacterial classes with less than $0.5 \%$ of total abundance. (f) Results of LEfSe analysis showing bacteria, at the lowest taxonomic level, that were significantly different in abundance in centenarians (Ce; $\mathrm{n}=17$ individuals) vs healthy controls (HC; $\mathrm{n}=14$ individuals). $\mathrm{p}$ : phylum; c: class; o: order; f: family; g: genus.

Figure 3. Effects of fecal microbiota transplantation in progeroid and WT mice (a) Scheme of the experimental design, in which the effects of FMT were assessed using 4 different groups of progeroid mice: control untransplanted $L m n a^{G 609 G / G 609 G}$ mice $(\mathrm{n}=11)$, $L_{m n a}{ }^{G 609 G / G 609 G}$ mice transplanted with fecal microbiota from WT mice $\left(L^{2 m n a^{G 609 G / G 609 G}}\right.$ WTmic; $\mathrm{n}=11$ ), Lmna ${ }^{G 609 G / G 609 G}$ mice transplanted with microbiota from older Lmna ${ }^{G 609 G / G 609 G}$ mice $\left(L m n a^{G 609 G}-0 G 609 G m i c ; n=11\right)$ and Lmna ${ }^{G 609 G / G 609 G}$ mice subjected to ablation of their own microbiota and transplanted with empty buffer (Lmna ${ }^{G 609 G}$-EmptyT; $\mathrm{n}=8$ ). Transplants were carried on at $\sim 8-10$ weeks of age, using as donors 4 months-old WT and 4 months-old Lmna ${ }^{G 609 G / G 609 G}$ mice. (b) Comparison of the percentage of initial body weight between $L m n a^{G 609 G / G 609 G}(\mathrm{n}=11)$ and $L m n a^{G 609 G / G 609 G_{-}}$ WTmic $(\mathrm{n}=11)$ mice over the indicated time period. Differences in body weight over 
time were assessed with a linear mixed model and analyzed with an Anova Type II Wald

489 Chi-square test $\left(X^{2}=8.06, \mathrm{df}=1, P=0.0045\right)$. Data are presented as mean $\pm \mathrm{SEM}$. (c)

490 Box plots showing differences in the body temperature between WT $(n=4)$, 491 Lmna $^{G 609 G / G 609 G}(\mathrm{n}=6)$ and Lmna ${ }^{G 609 G / G 609 G}$-WTmic $(\mathrm{n}=5)$ mice. One-way ANOVA 492 with Tukey's correction $(\mathrm{F}=20.45, \mathrm{df}=2, P=0.0001)$. Exact adjusted $\mathrm{p}$-values are 493 reported within the plot. (d) Comparison of the glucose levels between Lmna ${ }^{G 609 G / G 609 G}$ $494(\mathrm{n}=6)$ and Lmna ${ }^{G 609 G / G 609 G_{-}}$WTmic $(\mathrm{n}=5)$ mice over the indicated time period. Unpaired 495 two-tailed Student's $t$-test (for week 5: $\mathrm{t}=2.74$, df $=36, P=0.009$ ). (e) Representative 496 histological images of renal vasculature, showing increased perivascular fibrosis in 497 Lmna ${ }^{G 609 G / G 609 G}$ mice $(\mathrm{n}=10)$ (blue staining indicated with an arrow) compared to WT 498 ( $\mathrm{n}=10)$ and $L m n a^{G 609 G / G 609 G}$ mice transplanted with WT microbiota (Lmna ${ }^{G 609 G}$-WTmic; $499 \mathrm{n}=8$ ). Renal fibrosis scores were analyzed with a Kruskall-Wallis test with Dunn's 500 correction. Exact adjusted p-values are reported within the plot. Each dot represents a 501 single mouse. The horizontal line represents the mean $\pm 95 \%$ of confidence interval (CI). 502 Scale bar $=100 \mu \mathrm{m}$. (f) Percentage survival of Lmna ${ }^{G 609 G / G 609 G}(\mathrm{n}=11)$, Lmna $^{G 609 G / G 609 G_{-}}$ 503 WTmic $(\mathrm{n}=11)$ and Lmna ${ }^{G 609 G / G 609 G}$ mice transplanted with old Lmna ${ }^{G 609 G / G 609 G}$ 504 microbiota $\left(\right.$ Lmna $^{G 609 G}$-oG609Gmic; $\left.\mathrm{n}=11\right)$. Differences were analyzed with the Log505 rank Mantel-Cox test and $\mathrm{BH}$ correction was applied after pairwise comparisons between all experimental groups, including Empty transplant (Extended Data Fig. 6f). Hazard ratio (HR) was calculated using a Cox proportional model. For Lmna ${ }^{G 609 G / G 609 G}$-WTmic vs Lmna ${ }^{G 609 G / G 609 G}$, HR of 0.2 [95\% confidence interval (CI) 0.07-0.53], $P=0.0012$; for $L^{2 m n a^{G 609 G / G 609 G}}{ }_{-o G 609 G m i c}$ vs Lmna ${ }^{G 609 G / G 609 G}$, HR of 4.1 [95\% CI 1.5-11.1], P =

510 0.005. Median and maximal survival, percentage of median and maximal lifespan 511 extension and log-rank test adjusted p-values are indicated in the Kaplan-Meier plot. 512 Transplantation was performed starting at $\sim 8-10$ weeks of age. (g) Comparison of body 513 weight between male WT mice transplanted with progeroid microbiota (WT514 oG609Gmic; $n=7)$ and male WT controls $(n=7)$ over the indicated time period. 515 Differences of body weight over time were assessed with a linear mixed model and 516 analyzed with an Anova Type II Wald Chi-square test $\left(X^{2}=5.49\right.$, df $\left.=1, P=0.019\right)$. Data 517 is presented as mean \pm SEM. (h) Blood glucose levels of WT mice ( $\mathrm{n}=14 ; 7$ males and 5187 females) and WT-oG609Gmic $(\mathrm{n}=14 ; 7$ males and 7 females $)$. Unpaired two-tailed 519 Student's $t$-test $(\mathrm{t}=4.56, \mathrm{df}=26, P=0.0001)$. Each dot represents a single mouse. The 520 horizontal line represents the mean $\pm 95 \%$ CI. (i-k) Metabolic parameters, measured with 
an Oxymax system, of WT ( $\mathrm{n}=14 ; 7$ males and 7 females $)$ and WT-oG609Gmic $(\mathrm{n}=14$;

5227 males and 7 females) mice. Differences were analyzed with an unpaired two-tailed

523 Welch's $t$-test. (i) Volume of $\mathrm{O}_{2}$ consumed $\left(\mathrm{VO}_{2}\right)(\mathrm{t}=2.71, \mathrm{df}=28, P=0.011)$. (j)

524 Volume of $\mathrm{CO}_{2}$ produced $\left(\mathrm{VCO}_{2}\right)(\mathrm{t}=3.94, \mathrm{df}=23, P=0.0006)$. (k) Energy expenditure $525(\mathrm{EE})(\mathrm{t}=3.15, \mathrm{df}=25, P=0.0043)$. (l) Representative pictures of control Zmpste $24^{-/}$ 526 mice and Zmpste24\% mice transplanted with WT microbiota (Zmpste $24^{-/}$-WTmic). 527 Transplanted mice appear to be healthier, as manifested by an ameliorated cervicothoracic 528 lordokyphosis, a larger size and better grooming. $(\mathbf{m})$ Body weight at 35 weeks of life of 529 Zmpste $24^{--}$mice $(\mathrm{n}=4)$ and Zmpste $24^{-/}-\mathrm{WTmic}$ mice $(\mathrm{n}=6)$. Unpaired two-tailed 530 Student's $t$-test $(\mathrm{t}=4.31, \mathrm{df}=8, P=0.0026)$. (n) Blood glucose levels of Zmpste $24^{-/}$mice $531(\mathrm{n}=5)$ and Zmpste $24^{-/}-$WTmic mice $(\mathrm{n}=7)$ at 30 weeks. Unpaired two-tailed Student's $532 t$-test $(\mathrm{t}=5.47, \mathrm{df}=11, P=0.0002)$. (o) Percentage survival of Zmpste $24^{-/}(\mathrm{n}=7)$ and 533 Zmpste $24^{--}$-WTmic female mice $(\mathrm{n}=7)$. Differences were performed with the Log-rank 534 Mantel-Cox test $(P=0.0092)$. HR of 0.15 [95\% CI 0.03-0.75], $P=0.021$. Median and 535 maximal survival, percentage of median and maximal lifespan extension and exact p536 value are indicated in the Kaplan-Meier plot. Transplantation was performed starting at 537 7-10 weeks of age. For c, d, i-k, $\mathrm{m}$ and $\mathrm{n}$ : box plots show upper and lower hinges corresponding to the first and third quartiles, center line represents the median, whiskers indicate the highest and lowest values that are within $1.5 * \mathrm{IQR}$, and data beyond the end of the whiskers are outliers and plotted as points. Each dot represents a single mouse.

Figure 4. Akkermansia muciniphila supplementation in progeroid mice and metabolomic analysis of ileal content. (a) Percent survival of Lmna ${ }^{G 609 G / G 609 G}$ mice receiving A. muciniphila by oral gavage (AKK; $\mathrm{n}=9$ ) compared to Lmna ${ }^{G 609 G / G 609 G}$ mice (G609G; $\mathrm{n}=12$ ). A. muciniphila transplantation was performed starting at 7-8 weeks of age. Differences in survival were analyzed with Log-rank Mantel-Cox test $(P=0.0163)$. Hazard ratio (HR) was calculated using a Cox proportional model (HR of 0.31 [95\% confidence interval (CI) $0.11-0.86], P=0.0244$ ). (b) Relative expression levels of $\operatorname{Reg} 3 g$ and Tff3 in G609G $(\mathrm{n}=8)$ and AKK $(\mathrm{n}=7)$ mice. Differences were analyzed with an unpaired two-tailed Student's $t$-test. For Reg3g, t $=2.33$, df $=13, P=0.0365$; for Tff3, $\mathrm{t}$ $=2.42, \mathrm{df}=13, P=0.0306$. Data are represented as dots (one per mouse) with mean \pm 95\% of CI. (c) Left, representative histological images of the intestinal mucosa layer of G609G and AKK mice. Arrow indicates the thickness of the mucosa layer. Scale bar = 
$554100 \mu \mathrm{m}$. Right, comparison of mucosa layer thickness of G609G $(\mathrm{n}=8)$ and AKK $(\mathrm{n}=$

555 7) mice. Differences were calculated using an unpaired two-tailed Welch's $t$-test $(\mathrm{t}=2.72$,

$556 \mathrm{df}=12, P=0.018$ ). Data are represented as dots (one per mouse) with mean $\pm 95 \%$ of

557 CI. For b and c, G609G group was composed by 4 males and 4 females, and AKK group

558 was composed by 4 males and 3 females. (d) Metabolic set enrichment analysis of all

559 metabolites with statistically significant differences between the three groups: WT $(\mathrm{n}=$

560 8), $L m n a^{G 609 G / G 609}(\mathrm{n}=8)$ and $L m n a^{G 609 G / G 609 G}$-WTmic $(\mathrm{n}=8)$, using all annotated KEGG

561 pathways (see Methods). (e) Box plots showing the relative levels of different bile acids

562 in WT ( $=8)$, Lmna $^{G 609 G / G 609}(\mathrm{n}=8)$, and Lmna ${ }^{G 609 G / G 609 G}$-WTmic $(\mathrm{n}=8)$. CA: cholic

563 acid; CDCA: chenodeoxycholic acid; $\beta$ MCA: beta-muricholic acid; DCA: deoxycholic

564 acid; HCA: hyocholic acid; $\omega$ MCA: omega-muricholic acid; UDCA: ursodeoxycholic

565 acid; 12-KCDCA: 12-ketochenodeoxycholic acid. (f) Box plots showing the relative

566 levels of selected metabolites between WT $(\mathrm{n}=8), L m n a^{G 609 G / G 609 G}(\mathrm{n}=8)$ and

$567 L m n a^{G 609 G / G 609 G}$-WTmic $(\mathrm{n}=8)$. For e and $\mathrm{f}$, each group of 8 mice was composed by 4

568 males and 4 females. In both panels, differences were analyzed using a one-way ANOVA

569 with multiple comparison test with one-side relative to control Lmna ${ }^{G 609 G / G 609 G}$ mice.

570 Exact adjusted p-values are reported in each plot. In the box plots, upper and lower hinges

571 correspond to the first and third quartiles, center line represents the median, whiskers

572 indicate the highest and lowest values that are within $1.5 * \mathrm{IQR}$, and data beyond the end

573 of the whiskers are outliers and plotted as points. Each dot represents a single mouse. (g)

574 Schematic representation of the model proposed in this work. WT mice are characterized

575 by a gut symbiosis in which bacterial metabolites participate in systemic homeostasis. By

576 contrast, progeroid mice are characterized by a gut dysbiosis, leading to decreased bile

577 acid levels and a reduced healthspan and lifespan. FMT with WT microbiota (WT mic)

578 into progeroid mice raises bile acids levels and improves healthspan and lifespan.

579

580 
Mouse models

583

Both Lmna $6609 G / G 609 G$ and Zmpste24\% mice were generated by crossing

$584 \mathrm{Lmna}^{\mathrm{G609G/+}}$ and Zmpste $24^{+/-}$mice and genotyped in our laboratory as previously

585 described ${ }^{16,21}$. All mice used in this study were in C57BL/6N background. Mice were

586 caged separately by sex and transplantation group and checked daily for water and food

587 availability, as well as for good physical condition. Mice were housed in cages with solid

588 floors, sawdust and nests. Mice in all groups were given every day pellets of food

589 previously softened in water for 1-2 hours to facilitate the feeding of progeroid mice. All

590 components of the cages, including food, had been previously autoclaved. For glucose

591 determination, blood samples were obtained from the tail vein and measured with Accu-

592 Chek glucometer (Roche Diagnostics; Mannheim, Germany). Body temperature was

593 measured by rectal probe (Acorn ${ }^{\circledR}$ Temp TC Thermocouple Thermometer, Fisher

594 Scientific; Hampton, NH). Transplantation experiments in progeroid mice began at 6-

59510 weeks of age. In the $L m n a^{G 609 G / G 609 G}$ survival experiments, 11 mice were analyzed in

596 control (7 males and 4 females), transplanted with WT (5 males and 6 females) and

597 transplanted with Lmna ${ }^{G 609 G / G 609 G}$ groups (5 males and 6 females), whereas 8 mice (3

598 males and 5 females) were analyzed in the empty transplant group. Zmpste $24^{-/}$

599 transplantation experiments were performed with 7 females per group. In the WT

600 transplantation experiments, 14 animals per group were used (7 males and 7 females). In

601 the WT and Lmna ${ }^{G 609 G / G 609 G}$ validation experiment, 8 mice (4 males and 4 females) were

602 used in each group of transplanted mice, and pooled samples from 12 WT (6 males and 6

603 females) and 15 Lmna $^{G 609 G / G 609 G}$ mice (8 males and 7 females) were used as donors.

604 Survival curves were analyzed with Log-rank (Mantel-Cox) test. Maximum survival was 
606 by the Committee for Animal Experimentation of the Universidad de Oviedo (Spain) and

607 performed in accordance with the European and Spanish legislative and regulatory 608 guidelines (European convention ETS 123 , on the use and protection of vertebrate

609 mammals in experimentation and for other scientific purposes, and Spanish Law 6/2013,

610 and R.D. 53/2013 on the protection and use of animals in scientific research), making

611 every effort to minimize mouse discomfort.

\section{Human samples}

613 We obtained samples from four HGPS families and one NGPS family that include 614 individuals affected with progeria and their healthy siblings. Additionally, we collected

615 samples from a Spanish cohort composed by 17 centenarians, independently of their 616 health status, and 14 healthy ethnically-matched adults, aged 30-50 years and with no 617 history of any major disease. Research involving humans was approved by the Ethical 618 Committee of Regional Clinical Research of the Principality of Asturias, project 619 no.105/16. All participants read and signed an informed consent. extraction. For human studies, samples were picked and stored at $-20^{\circ} \mathrm{C}$ in OmniGene

623 Gut kits (Ref. OMR-200, DNA Genotek; Ora Sure Techonologies; Bethlehem, PN). In 624 all cases (mice and human samples), DNA was extracted using the PowerSoil DNA 625 Isolation kit (MO BIO Laboratories, Quiagen N.V.; Hilden, Germany). DNA quality and 626 quantification were assessed with Qubit fluorometer (Thermo Fisher Scientific; Waltham, 627 MA). Libraries were prepared following the 16S Metagenomic Sequencing Library 628 Preparation protocol from Illumina. Briefly, the region V3-V4 from 16S rRNA was amplified using the primers 341F/805R to which Illumina Sequencing adapters and dual- 
630 index barcodes of the Nextera XT kit were added (FWD 5'-

631 TCGTCGGCAGCGTCAGATGTGTATAAG AGACAGCCTACGGGNGGCWGCAG-

632 3' and REV 5'-GTCTCGTGGGCTCGGAGA

633 TGTGtATAAGAGACAGGACTACHVGGGTATCTAATCC-3'). Sequencing was

634 performed in a MiSeq platform (IMEGEN, Valencia, Spain) using 2 x 300 bp-end

635 protocol.

636 Metagenome profiling

637 Raw paired-end reads were processed with QIIME 2 (version 2018.6.0). Sequence

638 quality controls were performed with DADA2 (qiime dada2 denoise-paired, with a

639 number of expected errors higher than 6): reads were filtered, trimmed, denoised,

640 dereplicated, forward and reverse sequences were merged, and chimeras were removed.

641 Taxonomy was assigned using a pre-trained Naïve Bayes classifier, with a trimmed

642 version of Greengenes 13_8 99\% OTUs, which includes the V3-V4 regions, bounded by

643 the 341F/805R primer pair. For $L m n a^{G 609 G / G 609 G}$ model, we obtained 3,071,354 paired-

644 end reads of 300 nucleotides each, with 1,084 OTUs identified after quality filtering

645 (Extended Data Fig 1a), and for Zmpste24 $4^{-/}$model, we obtained 1,149,187 paired-end

646 reads of 300 nucleotides each, with 882 OTUs identified after quality-filtering (Extended

647 Data Fig 3a). 4-month-old C57BL/6N WT mice in experiments from Figure 1 where used

648 as controls for both $L m n a^{G 609 G / G 609 G}$ and Zmpste $24^{-/-}$mice. For human samples in progeria

649 patients, we obtained 1,709,578 paired-end reads of 300 nucleotides each, with 691 OTUs

650 identified after quality filtering (Extended Data Fig 4a), whereas for centenarians and

651 healthy controls we obtained 6,196,891 paired-end reads of 300 nucleotides each, with

652 1,761 OTUs identified after quality-filtering (Extended Data Fig 5a). For validating FMT

653 experiments 8 mice per group and condition were used, obtaining 8,761,200 paired-end

654 reads of 300 nucleotides each, with 18,402 OTUs identified after quality filtering. Alpha 
655 diversity and statistical analysis were calculated based on different metrics (shannon,

656 chao1). Beta diversity was measured using Bray-Curtis dissimilarity and Jaccard 657 similarity index (braycurtis, jaccard). Samples were hierarchical clustered with the

658 Unweighted Pair Group Method with Arithmetic Mean (UPGMA) method and using 659 different beta diversity metrics (braycurtis, jaccard). Differences in bacteria abundance 660 were calculated using LEfSe ${ }^{42}$. Metagenome functional content prediction was performed 661 using PICRUSt ${ }^{43}$ and HUMAnN2 (v0.11.1) ${ }^{44}$, and analyzed with LEfSe.

662

663

664

665

666

667

668

669

670

671

672

673

674

\section{Microbiome transplants}

Prior to transplantation, mice were treated for three consecutive days with $200 \mu \mathrm{L}$ of an antibiotic-cocktail (with each daily dose being administered by oral gavage after a 6-hour fast) which contained $1 \mathrm{~g} / \mathrm{L}$ ampicillin, $0.5 \mathrm{~g} / \mathrm{L}$ neomycin, $0.5 \mathrm{~g} / \mathrm{L}$ vancomycin and $1 \mathrm{~g} / \mathrm{L}$ metronidazole. Thereafter mice were given $100 \mu \mathrm{L}$ of the microbiome suspension twice a week for 2 weeks, starting the first day after the antibiotic cycle. After this 2-week period mice received the microbiome suspension once a week until natural death or sacrifice. For the microbiome suspension preparation, 2-5 fresh feces pellets (80-100 $\mathrm{mg}$ ) were resuspended with a vortex in $600 \mu \mathrm{L}$ of reduced PBS (PBS with $0.5 \mathrm{~g} / \mathrm{L}$ cysteine and $0.2 \mathrm{~g} / \mathrm{L} \mathrm{Na} \mathrm{N}_{2} \mathrm{~S}$ ). After resuspension, tubes containing the feces in reduced PBS were centrifuged at 2,500 rpm (500 g) for $1 \mathrm{~min}$ to remove insolubilized material, and $100 \mu \mathrm{L}$ of supernatant were administered to the mice by oral gavage. Empty transplant group received the same antibiotics treatment and were transplanted only with reduced PBS.

\section{Calorimetry measurements}

Metabolic parameters (oxygen consumption, carbon dioxide production and total energy expenditure), were obtained using the comprehensive lab animal monitoring system (Oxymax CLAMS, Columbus Instruments; Columbus, OH) and analyzed 
679 following the manufacturer's instructions. Mice were monitored for $48 \mathrm{~h}$ and the first 24

$680 \mathrm{~h}$ were discarded in the analysis, considering them as acclimation period.

\section{Akkermansia muciniphila culture and oral supplementation}

Cultures of the strain Akkermansia muciniphila CIP107961 grown for $24 \mathrm{~h}$ in

683 GAM medium (Nissui Pharmaceutical Co; Tokyo, Japan) supplemented with $0.25 \%$

684 (w/v) L-cysteine (Sigma Chemical Co.; St. Louis, MO) (GAMc) in anaerobic conditions

685 were used to inoculate $(2 \% \mathrm{v} / \mathrm{v})$ fresh pre-reduced GAMc broth which was incubated for

$68624 \mathrm{~h}$. Afterwards, cultures were washed with PBS and concentrated in anaerobic PBS that

687 included $25 \%(\mathrm{v} / \mathrm{v})$ glycerol to a concentration of about $1 \times 10^{10} \mathrm{cfu} / \mathrm{mL}$ under strict

688 anaerobic conditions and stored at $-80{ }^{\circ} \mathrm{C}$ until use. To test the viability of glycerol

689 stocks, serial dilutions in PBS were made and deep plated on agar-GAMc. Plates were

690 incubated under anaerobic conditions for 5 days to determine the Akkermansia counts

691 (cfu/ml). Before administration by oral gavage, the glycerol stocks were thawed under

692 anaerobic conditions and diluted with anaerobic PBS to a final concentration of $2 \times 10^{8}$

693 viable cfu/0.1 mL. Lmna ${ }^{G 609 G / G 609 G}$ mice were treated by oral gavage with $100 \mu \mathrm{L}$ of either

694 Akkermansia suspension (AKK group, $\mathrm{n}=9$ ) or anaerobic PBS (control group, $\mathrm{n}=12$ )

695 three days a week beginning at 12 weeks of age and until decease.

696

697

698

699

700

701

702

\section{Quantitative polymerase chain reaction}

For RNA expression analysis, total RNA from about $30 \mathrm{mg}$ of frozen ileon samples was extracted using Trizol (Life Technologies) and resuspended in nuclease-free water (Life Technologies). 1-2 $\mu$ g of total RNA was used for reverse transcription using the QuantiTect Reverse Transcription kit (Quiagen N.V.). 10× diluted cDNA was used for quantitative polymerase chain reaction (qPCR) using Power SYBR Green PCR Master Mix (Life Technologies; Carlsbad, CA) and Real-Time PCR (7300 HT, Applied 
703 Biosystems; Foster City, CA). Gene expression was normalized to the GAPDH 704 expression. For bacterial quantification, DNA from mouse feces was extracted as 705 described above. 1-4 ng of DNA were used for qPCR reactions using specific primers to 706 amplify bacterial 16S rDNA. Bacterial abundance was assessed by normalizing with the 707 abundance of total bacteria in feces using the conserved eubacterial 16S rDNA primer

708 pair UniF340/UniR514. Results are represented as relative quantification using RQ value $709\left(\mathrm{RQ}=2^{-\Delta \Delta C t}\right)$. Primer sets for qPCR analyses are shown in Supplementary Table 4.

Metabolomic analysis

Prior to sacrifice for sample collection, mice were starved overnight and thereafter allowed to eat for $4 \mathrm{~h} .30 \mathrm{mg}$ of ileum content for each condition were first weighted and solubilized into $1 \mathrm{~mL}$ polypropylene Precellys lysis tubes, with $1 \mathrm{~mL}$ of cold lysate buffer

$714\left(\mathrm{MeOH} / \mathrm{Water} / \mathrm{Chloroform}, 9 / 1 / 1,-20^{\circ} \mathrm{C}\right)$. After being vortexed for $10 \mathrm{~min}$, samples were 715 centrifuged $\left(10 \mathrm{~min}\right.$ at $\left.15,000 \mathrm{~g}, 4^{\circ} \mathrm{C}\right)$, and the upper phase was collected and split in two 716 parts: the first $270 \mu \mathrm{L}$ used for the Gas Chromatography coupled to Mass Spectrometry 717 (GC-MS) and $250 \mu \mathrm{L}$ used for the Ultra High Pressure Liquid Chromatography coupled to Mass Spectrometry (UHPLC-MS). For GC-MS measurements, $150 \mu \mathrm{L}$ from the

719 aliquot were transferred to a glass tube and evaporated. Then, $50 \mu \mathrm{L}$ of methoxyamine

720 (20 mg/mL in pyridine) was added to dried extracts and samples were then stored at room temperature in the dark for $16 \mathrm{~h}$. The day after, $80 \mu \mathrm{L}$ of MSTFA was added and final derivatization carried out at $40{ }^{\circ} \mathrm{C}$ during $30 \mathrm{~min}$. Samples were then transferred in vials and directly injected into GC-MS. For LC-MS measurements, the collected supernatant was evaporated in microcentrifuge tubes at $40{ }^{\circ} \mathrm{C}$ in a pneumatically-assisted concentrator

725 (Techne DB3, Techne; Staffordshire, UK). Dried extracts were solubilized with $450 \mu \mathrm{L}$ of MilliQ water and aliquoted in 3 microcentrifuge tubes $(100 \mu \mathrm{L})$ for each LC method 
and one microcentrifuge tube for safety. Aliquots were transferred to LC vials and

728 injected into LC-MS or kept at $-80{ }^{\circ} \mathrm{C}$ until injection. A daily qualification of the instrumentation was set up with automatic tune and calibration processes. These

730 qualifications were completed with double injections of standards mixes, at the beginning 731 and at the end of the run, as for a blank extracted sample to control the background 732 impurities. Mixtures were adapted for each chromatographic method. After the 733 extraction, fractions of each biological sample were pooled to create a Quality Control 734 (QC) sample, use to passivate the column before the analysis with the proper biological matrix. This QC sample was re-injected in each batch to monitor and correct analytical bias. Analytical methods and data processing were performed as previously described ${ }^{45}$.

737 Results were represented as the normalized area of the MS picks in $\log 2$ scale using 738 arbitrary units. Normalization was performed by correcting the area of the MS picks across the batches using the QC pooled samples and by centering their values around the mean of the QC areas. Standard reagents (acetonitrile, methanol, chloroform, acetic acid and dibutylamine acetate concentrate) were acquired from Sigma Aldrich (Saint Luis,

742 MO). Differentially expressed metabolites in each condition were identified using 743 moderate t-statistic implemented in the R/Bioconductor package limma ${ }^{46}$, using sex as a covariate. Metabolites with a nominal $P$-value $<0.05$ and q-value $<0.25$ were selected for metabolic set enrichment analysis using one-sided Fisher's exact test against all metabolites annotated in each KEGG pathway. Metabolomic results are provided in

747 Supplementary Table 5.

Histological analysis

Kidneys and intestines were fixed in 4\% paraformaldehyde in PBS and stored in

$75050 \%$ ethanol. Fixed tissues were embedded in paraffin by standard procedures. Blocks were sectioned $(5 \mu \mathrm{m})$ and stained with hematoxylin and eosin and Masson trichrome 
752 (H\&E, MT, kidney) and periodic acid Schiff-alcian blue (PAS-AB, intestine). Renal 753 perivascular fibrosis was analyzed/graded from 0 to 4 , by using a histology damage score

754 (0: no lesion; 1: focal lesion; 2: multifocal mild lesion; 3: multifocal moderate lesion; 4:

755 diffuse, moderate or severe damage). Five fields were scored from each slide.

756

757

758

759

760

761

762

763

764

765

766

767

768

769

770

771

772

773

774

\section{Statistical analysis}

Number of mice allocated per group was based on previous experiments and their distribution was randomized, being indicated in each Figure legend. Comparisons between two groups following normal distribution were performed using a two-tailed Student's $t$-test, while one-factor analysis of variance (ANOVA) was used for comparisons of three or more groups. Unless specified in the Figure legends, adjusted $p$ values were obtained using Tukey's correction. For non-parametric distributions, the Wilcoxon rank-sum and Kruskal-Wallis test (the latter followed by the Dunnett post-hoc test) were performed for comparisons between two groups or three or more groups, respectively. Survival analysis was performed by using the Kaplan-Meier method and statistical differences were analyzed with the Log-rank (Mantel-Cox) test (GraphPad Prism 6.0 and survival R package). Body weight curves were analyzed using a linearmixed effect model (lme4 $\mathrm{R}$ package). The hazard ratio was calculated using a Cox proportional hazards regression model (survival R package). Sample sizes for lifespan experiments were chosen with a power of $80 \%$, based on our previous studies ${ }^{16,28,47}$. Maximal survival was calculated using Fisher's test at $80^{\text {th }}$ percentile. Plots were generated with GraphPad Prism 6.0 and RStudio (using ggplot2 R package), and edited with Illustrator CC (21.0.0). Statistical analysis was performed using RStudio and GraphPad Prism 6.0. Exact P-values are indicated in each figure. 
777 Sciences Reporting Summary linked to this article.

\section{Data availability statement}

780 Sequence data supporting these findings have been deposited in EGA under with accession number EGAS00001003656. Metabolomics data are provided in the Supplementary Table 5. Any additional data generated and analyzed in this study are available from the corresponding authors upon reasonable request.

Methods-only references

786

41. Wang, C., Li, Q., Redden, D.T., Weindruch, R. \& Allison, D.B. Statistical methods for testing effects on "maximum lifespan". Mech Ageing Dev 125, 629-632

789 (2004).

42. Segata, N., et al. Metagenomic biomarker discovery and explanation. Genome Biol 12, R60 (2011).

43. Langille, M.G., et al. Predictive functional profiling of microbial communities using 16S rRNA marker gene sequences. Nat Biotechnol 31, 814-821 (2013).

44. Abubucker, S., et al. Metabolic reconstruction for metagenomic data and its application to the human microbiome. PLoS Comput Biol 8, e1002358 (2012). depleted by palmitate but increased by oleate in vivo. Cell cycle 14, 2399-2407 (2015).

46. Ritchie, M.E., et al. limma powers differential expression analyses for RNAsequencing and microarray studies. Nucleic acids research 43, e47 (2015).

800 47. Osorio, F.G., et al. Nuclear lamina defects cause ATM-dependent NF-kappaB 801 activation and link accelerated aging to a systemic inflammatory response. Genes Dev 26, 2311-2324 (2012). 